Case Report

\title{
Bilateral Renal Tumour as Indicator for Birt-Hogg-Dubé Syndrome
}

\author{
P. C. Johannesma, ${ }^{1}$ R. J. A. van Moorselaar, ${ }^{2}$ S. Horenblas, ${ }^{3}$ L. E. van der Kolk, ${ }^{4}$ \\ E. Thunnissen, ${ }^{5}$ J. H. T. M. van Waesberghe, ${ }^{6}$ F. H. Menko, ${ }^{4,7}$ and P. E. Postmus ${ }^{1}$ \\ ${ }^{1}$ Department of Pulmonary Diseases, VU University Medical Center, P.O. Box 7057, 1007 MB Amsterdam, The Netherlands \\ ${ }^{2}$ Department of Urology, VU University Medical Center, Amsterdam, The Netherlands \\ ${ }^{3}$ Urologic Oncology and Department of Urology, The Netherlands Cancer Institute, P.O. Box 90203, \\ 1006 BE Amsterdam, The Netherlands \\ ${ }^{4}$ Department of Clinical Genetics, The Netherlands Cancer Institute, P.O. Box 90203, 1006 BE Amsterdam, The Netherlands \\ ${ }^{5}$ Department of Pathology, VU University Medical Center, Amsterdam, The Netherlands \\ ${ }^{6}$ Department of Radiology, VU University Medical Center, Amsterdam, The Netherlands \\ ${ }^{7}$ Department of Clinical Genetics, VU University Medical Center, Amsterdam, The Netherlands
}

Correspondence should be addressed to P. E. Postmus; pe.postmus@vumc.nl

Received 14 January 2014; Accepted 21 February 2014; Published 20 March 2014

Academic Editor: Gottfried J. Locker

Copyright ( 2014 P. C. Johannesma et al. This is an open access article distributed under the Creative Commons Attribution License, which permits unrestricted use, distribution, and reproduction in any medium, provided the original work is properly cited.

Birt-Hogg-Dubé (BHD) syndrome is a cancer disorder caused by a pathogenic FLCN mutation characterized by fibrofolliculomas, lung cysts, pneumothorax, benign renal cyst, and renal cell carcinoma (RCC). In this case we describe a patient with bilateral renal tumour and a positive familial history for pneumothorax and renal cancer. Based on this clinical presentation, the patient was suspected for BHD syndrome, which was confirmed after molecular testing. We discuss the importance of recognizing this autosomal dominant cancer disorder when a patient is presented at the urologist with a positive family history of chromophobe renal cell cancer or a positive familial history for renal cell cancer and pneumothorax.

\section{Background}

Birt-Hogg-Dubé syndrome (BHDS) was originally described in 1977 and is nowadays known as a rare autosomal dominant cancer disorder characterized by fibrofolliculomas, lung cysts, pneumothorax, benign renal cyst, and renal cell carcinoma (RCC). The mutated gene for BHD encodes the protein folliculin $(F L C N)$ which acts as a tumour suppressor and interacts with mTOR and AMPK signalling pathways [1]. Here we report a case of a patient with bilateral renal cancer and a positive familial history for pneumothorax and renal cancer. Based on the bilateral renal tumour and the positive family history for renal cancer and pneumothorax, Birt-Hogg-Dubé syndrome was suspected.

\section{Case Report}

In March 2011, a 44-year-old Caucasian male was evaluated for urolithiasis. He had no physical complaints, macroscopic haematuria, or weight loss. His medical and social history were unremarkable; he never smoked. His father had been treated for colorectal cancer; his mother had three episodes of spontaneous pneumothorax and had been treated for a renal tumour. Physical examination of the abdomen showed no abnormalities. Routine laboratory tests were normal. Computed tomography (CT) of the abdomen showed an interpolar tumour in the left kidney, diameter $14 \mathrm{~mm}$ (Figure 1(a), arrow), and a second tumour in the upper pole of the right kidney, diameter $8 \mathrm{~mm}$ (Figure 1(b), arrow). After a needle biopsy of the largest tumour, revealing a chromophobe renal cell carcinoma (Figures 2(a) and 2(b)), the tumour in the left kidney was treated with radio frequency ablation (RFA). The CT findings in combination with the positive family history for renal cancer and his mother's episodes of pneumothorax suggested Birt-Hogg-Dubé (BHD) syndrome. Sequencing of the FLCN gene showed a pathogenic heterozygous frameshift mutation (c.155delc; p.Leu518Phefs $* 19$ ) - which has not been 


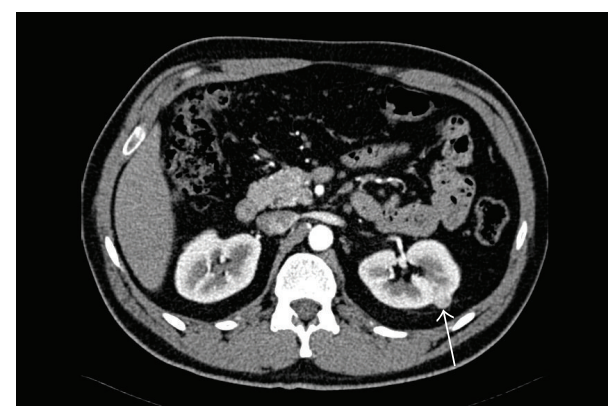

(a)

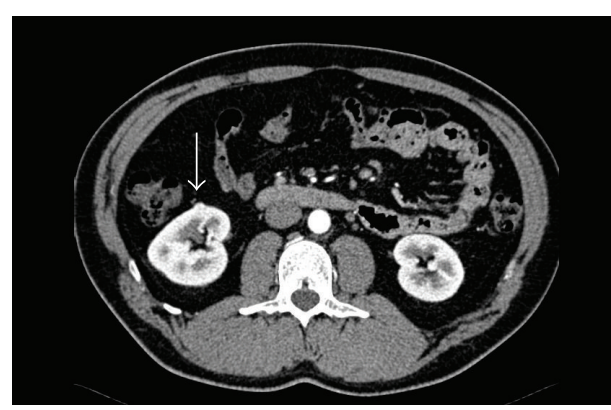

(b)

Figure 1: Contrast enhanced CT shows in the arterial phase a hypervascular small lesion in both kidneys, representing two small chromophobe renal cell carcinomas. The tumour in the left kidney (a) was treated by radiofrequency ablation (RFA); for the tumour in the right kidney, (b) follow up was proposed.

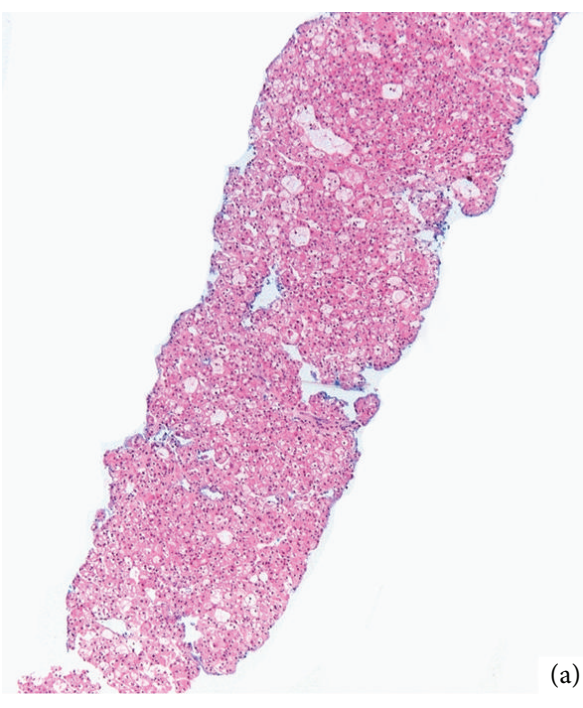

(a)

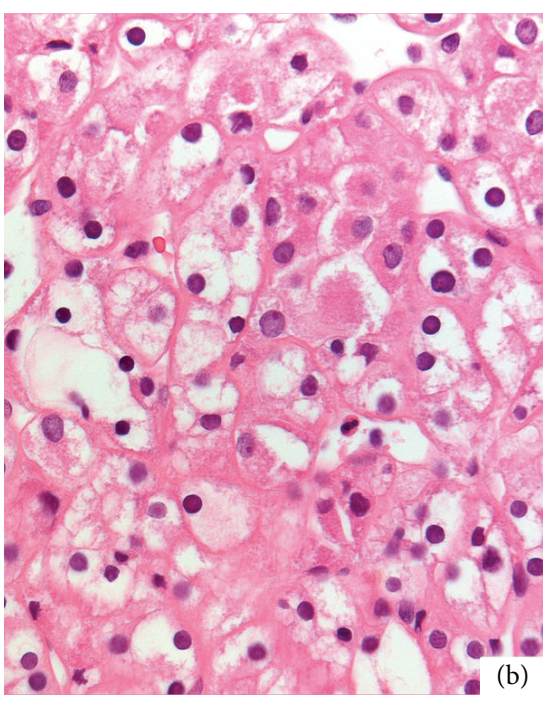

(b)

FigURE 2: Overview of needle biopsy of tumor in the left kidney ((a): amplification $\times 2.5)$ and detail ((b): amplification $\times 40)$ of a chromophobe renal carcinoma. Overview of needle biopsy $((\mathrm{a})$ : amplification $\times 2.5)$ and detail ((b): amplification $\times 40)$ of monotonous cellular pattern with mild nuclear pleomorphy and abundant partly eosinophilic cytoplasm with perinuclear halo, compatible with chromophobe renal cell carcinoma.

described before in literature-and confirmed the diagnosis of Birt-Hogg-Dubé syndrome. The index patient had neither siblings nor children. His parents died years ago; blood or tissue was not available for molecular testing. Frequent follow up by magnetic resonance imaging (MRI) will be performed for evaluation of the small tumour in the right kidney and possible recurrence of the tumour in the left kidney.

\section{Discussion and Conclusion}

Birt-Hogg-Dubé syndrome [OMIM \#135150] is a rare autosomal dominant inherited disorder caused by a mutation in the FLCN gene located on chromosome 17p11.2, which acts as a tumour suppressor and probably interacts with mTOR and AMPK signalling pathways [1]. BHD is clinically characterized by skin fibrofolliculomas, lung cysts, (recurrent) spontaneous pneumothorax, and renal cancer [2]. In literature, cooccurrence of $\mathrm{BHD}$ and a range of tumours, other than renal cancer, has been described, but so far a causal relationship between $\mathrm{BHD}$ and these benign and malignant tumours has not been proven [2]. Skin fibrofolliculomas are multiple, dome-shaped, whitish papules located on the scalp, forehead, face, and neck and are found in approximately $90 \%$ of families with confirmed BHD syndrome. Dermatologic consultation confirmed multiple fibrofolliculomas on the forehead and face of the index patient. Although cosmetic therapeutic options are limited, case reports suggest that laser ablation, using a YAG or fractional $\mathrm{CO}_{2}$ laser, gives temporary improvement [3].

BHD patients have a 50-fold higher risk to develop primary spontaneous pneumothorax (PSP) compared to the normal population. PSP in BHD patients occurs usually after the age of 20, although it has been described already at the age of 7 years. Up to $90 \%$ of BHD patients have multiple lung cysts, usually located in the basal regions [4]. In our clinic, we found an estimated penetrance for pneumothorax of $29 \%$ (CI: 
9-49\%) at 70 years of age. BHD patients usually have a normal lung function and no pulmonary symptoms. A CT of the chest performed in our index patient showed no pulmonary cysts.

Renal cell cancer (RCC) is the most lethal of the urologic malignancies, with estimated 273,518 new cases diagnosed and 116,368 patient deaths in 2008 worldwide [5].

RCC can be divided into sporadic and hereditary. The majority is of sporadic origin, presenting normally after the age of 60 as one lesion, while the hereditary type mainly presents as multifocal and bilateral at a far younger age. The most common hereditary renal cancer syndromes are associated with hereditary leiomyomatosis and renal cell carcinoma (HLRCC), Von Hippel-Lindau syndrome (VHL), and hereditary papillary renal carcinoma (HPRC). The prevalence of RCC in (familial) renal cancer is unknown and might be underestimated, since the majority (>80\%) of the BHD index patients are referred by a dermatologist.

In a large published series by Pavlovich et al., 34 of 124 individuals (27.4\%) with genetic confirmed BHD had a median of 5 renal tumours at a mean age of 50.4 years (range 31-74 years) [6]. In a large series published by Toro et al., $34 \%$ of individuals with BHD had renal tumours [4]. The youngest patient with BHD who developed renal cancer was 20 years old [7]. Another study reported a history of renal cancer and metastasis in the same year in a patient with BHD at age 27 [8].

In our Dutch study population published by Houweling et al., we found, among 115 BHD patients, 14 patients with renal cancer and calculated an estimated penetrance for renal cancer of 16\% (CI: 6-26\%) at 70 years of age [9]. Most lesions are a mixture of solid and cystic components. The largest published histological series of RCC among BHD patients demonstrates that these tumours contain both oncocytoma and chromophobe elements [10]. Houweling and colleagues confirmed these findings, as they found in the majority of RCC tumours cells with granular/floccular eosinophilic cytoplasm, as can be seen in both clear cell carcinoma and chromophobe carcinoma [9].

As the risk of developing RCC is high, imaging and follow up at regular intervals are advised by MRI from the age of 20. The role of ultrasound (US) for detecting renal tumours is still extensively discussed in literature. Surgical treatment is recommended before the largest tumour reaches $3 \mathrm{~cm}$ in maximal diameter, which is based on the VHL guideline [8]. Initially, a nephron-sparing surgery should be ideally pursued, which can help prevent chronic renal insufficiency in this patient population. Minimally invasive nephronsparing techniques such as cryoablation and radio frequency ablation (RFA) are generally accepted as treatment of choice in patients with a unifocal renal lesion. Since BHD patients are at lifelong risk for the development of new tumours, and cryoablation or RFA can complicate both the long term evaluation and surgical management, nephron-sparing surgery is so far the safest and most effective treatment for hereditary renal tumours [11].

In conclusion, in patients with a positive family history of chromophobe renal cell cancer or a positive family history for renal cell cancer and pneumothorax, the diagnosis of
BHD should be considered. Therefore, we suggest that easily accessible FLCN sequencing should be considered in patients and their families because of the high incidence of renal cancer in BHD patients.

\section{Abbreviations \\ FLCN: Folliculin \\ RCC: Renal cell carcinoma \\ BHD: Birt-Hogg-Dubé syndrome \\ CT: $\quad$ Computed tomography \\ RFA: Radio frequency ablation \\ mTOR: Mammalian target of rapamycin \\ AMPK: AMP-activated protein kinase \\ MRI: Magnetic resonance imaging \\ US: $\quad$ Ultrasound \\ PSP: $\quad$ Primary spontaneous pneumothorax \\ HLRCC: Hereditary leiomyomatosis and renal cell carcinoma \\ VHL: Von Hippel-Lindau syndrome \\ HPRC: Hereditary papillary renal carcinoma.}

\section{Ethical Approval}

In lieu of a formal ethics committee, the principles of the Helsinki Declaration were followed.

\section{Conflict of Interests}

The authors declare that they have no conflict of interests regarding the publication of this paper.

\section{Authors' Contribution}

P. C. Johannesma drafted the paper. S. Horenblas, L. E. van der Kolk, E. Thunnissen, and J. H. T. M. van Waesberghe provided the clinical data and gave critical comment on the paper. R. J. A. van Moorselaar, F. H. Menko, and P. E. Postmus supervised P. C. Johannesma while drafting the paper and wrote the final paper. All authors gave final approval of the paper.

\section{References}

[1] M. Baba, S.-B. Hong, N. Sharma et al., "Folliculin encoded by the BHD gene interacts with a binding protein, FNIP1, and AMPK, and is involved in AMPK and mTOR signaling," Proceedings of the National Academy of Sciences of the United States of America, vol. 103, no. 42, pp. 15552-15557, 2006.

[2] F. H. Menko, M. A. M. van Steensel, S. Giraud et al., "Birt-HoggDubé syndrome: diagnosis and management," The Lancet Onco$\log y$, vol. 10, no. 12, pp. 1199-1206, 2009.

[3] T. Gambichler, M. Wolter, P. Altmeyer, and K. Hoffman, "Treatment of Birt-Hogg-Dubé syndrome with erbium: YAG laser," Journal of the American Academy of Dermatology, vol. 43, no. 5, pp. 856-858, 2000.

[4] J. R. Toro, S. E. Pautler, L. Stewart et al., "Lung cysts, spontaneous pneumothorax, and genetic associations in 89 families with Birt-Hogg-Dubé syndrome," American Journal of Respiratory and Critical Care Medicine, vol. 175, no. 10, pp. 1044-1053, 2007. 
[5] J. Ferlay, H.-R. Shin, F. Bray, D. Forman, C. Mathers, and D. M. Parkin, "Estimates of worldwide burden of cancer in 2008: GLOBOCAN 2008," International Journal of Cancer, vol. 127, no. 12, pp. 2893-2917, 2010.

[6] C. P. Pavlovich, R. L. Grubb III, K. Hurley et al., "Evaluation and management of renal tumors in the Birt-Hogg-Dubé syndrome," The Journal of Urology, vol. 173, no. 5, pp. 1482-1486, 2005.

[7] S. K. Khoo, S. Giraud, K. Kahnoski et al., "Clinical and genetic studies of Birt-Hogg-Dubé syndrome," Journal of Medical Genetics, vol. 39, no. 12, pp. 906-912, 2002.

[8] I. Kluijt, D. de Jong, H. J. Teertstra et al., "Early onset of renal cancer in a family with Birt-Hogg-Dubé syndrome," Clinical Genetics, vol. 75, no. 6, pp. 537-543, 2009.

[9] A. C. Houweling, L. M. Gijezen, M. A. Jonker et al., "Renal cancer and pneumothorax risk in Birt-Hogg-Dubé syndrome; an analysis of 115 FLCN mutation carriers from 35 BHD families," British Journal of Cancer, vol. 105, no. 12, pp. 1912-1919, 2011.

[10] C. P. Pavlovich, M. M. Walther, R. A. Eyler et al., "Renal tumors in the Birt-Hogg-Dubé syndrome," The American Journal of Surgical Pathology, vol. 26, no. 12, pp. 1542-1552, 2002.

[11] L. Stamatakis, A. R. Metwalli, L. A. Middelton et al., "Diagnosis and management of BHD-associated kidney cancer," Familial Cancer, vol. 12, no. 3, pp. 397-402, 2013. 


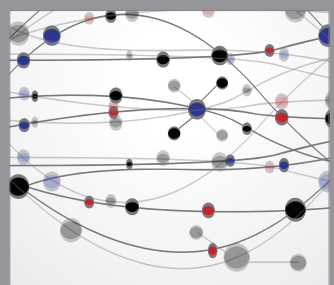

The Scientific World Journal
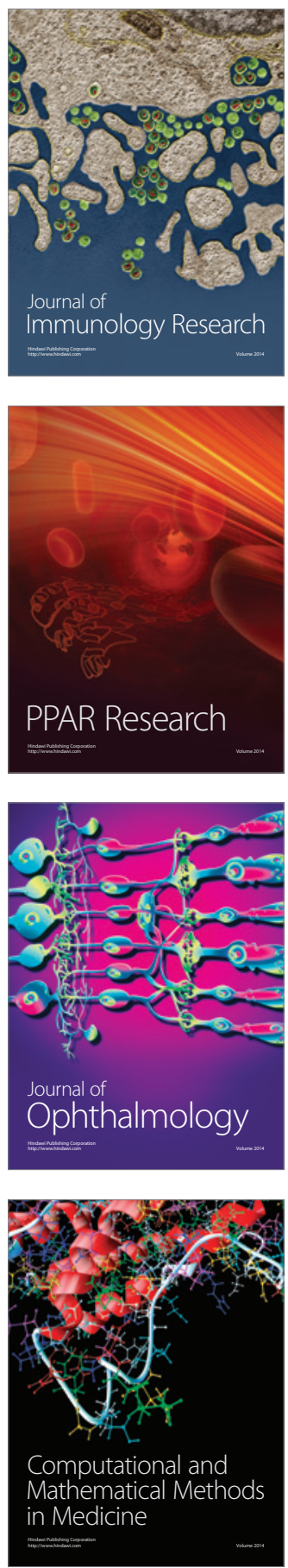

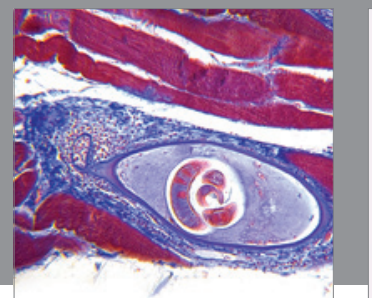

Gastroenterology

Research and Practice
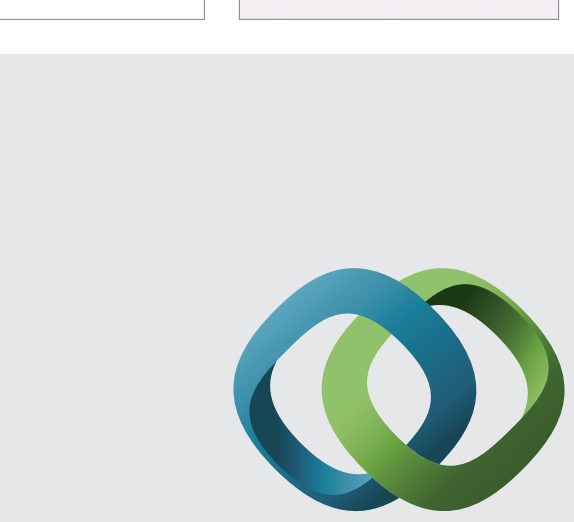

\section{Hindawi}

Submit your manuscripts at

http://www.hindawi.com
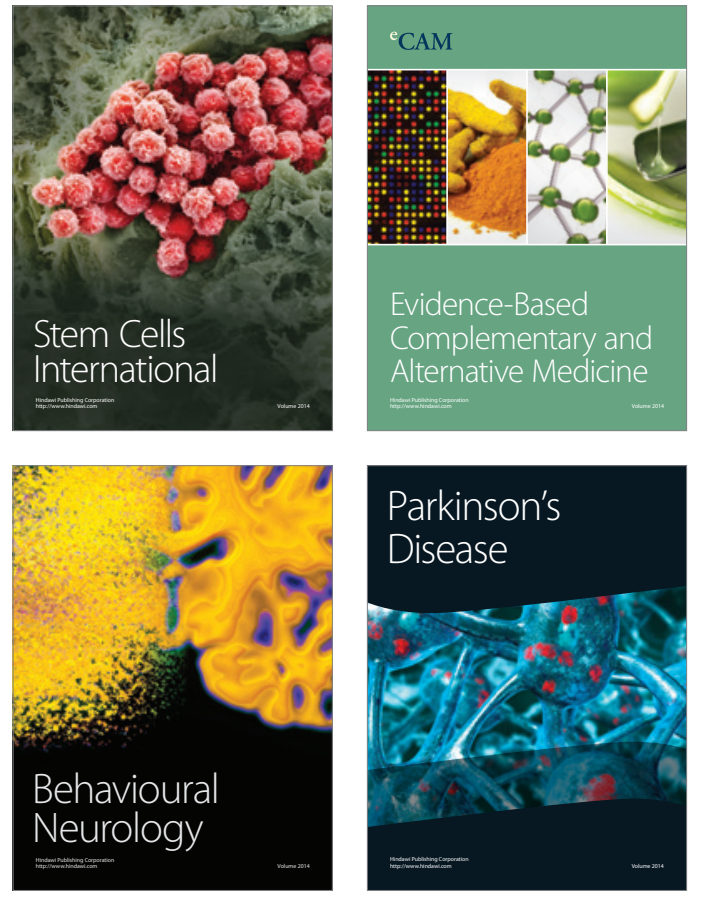
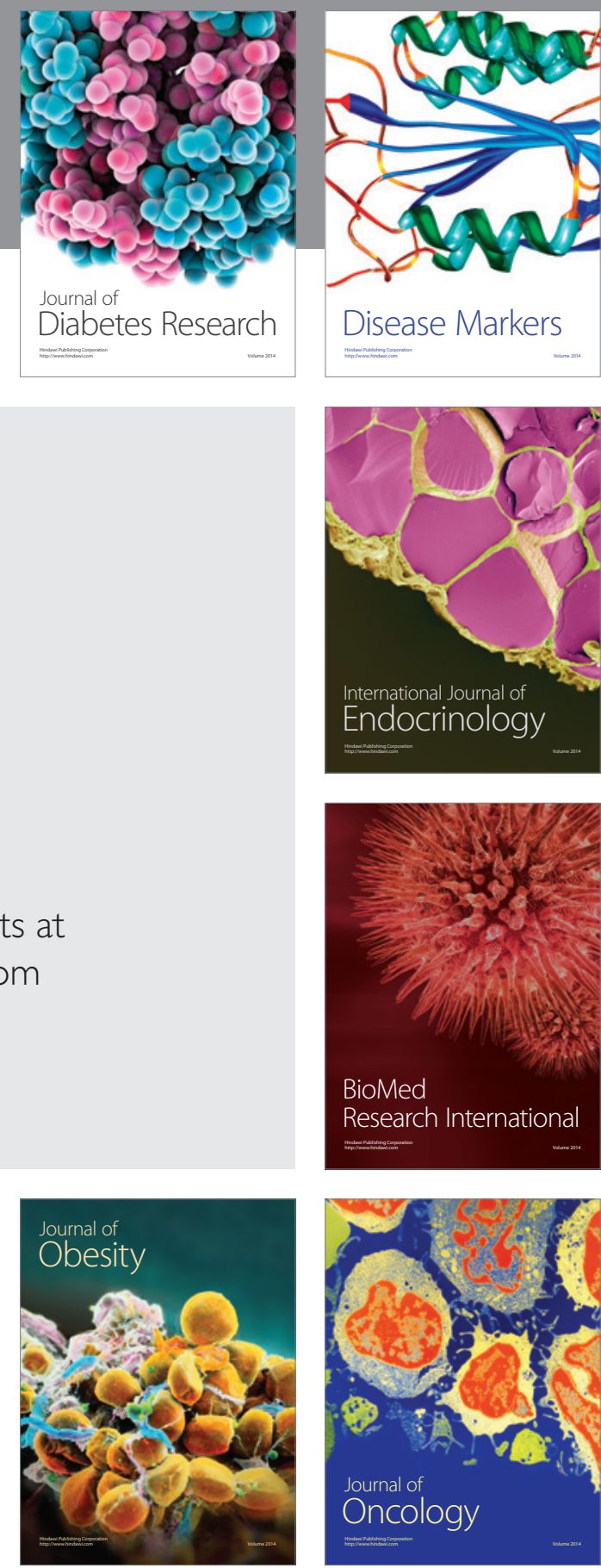

Disease Markers
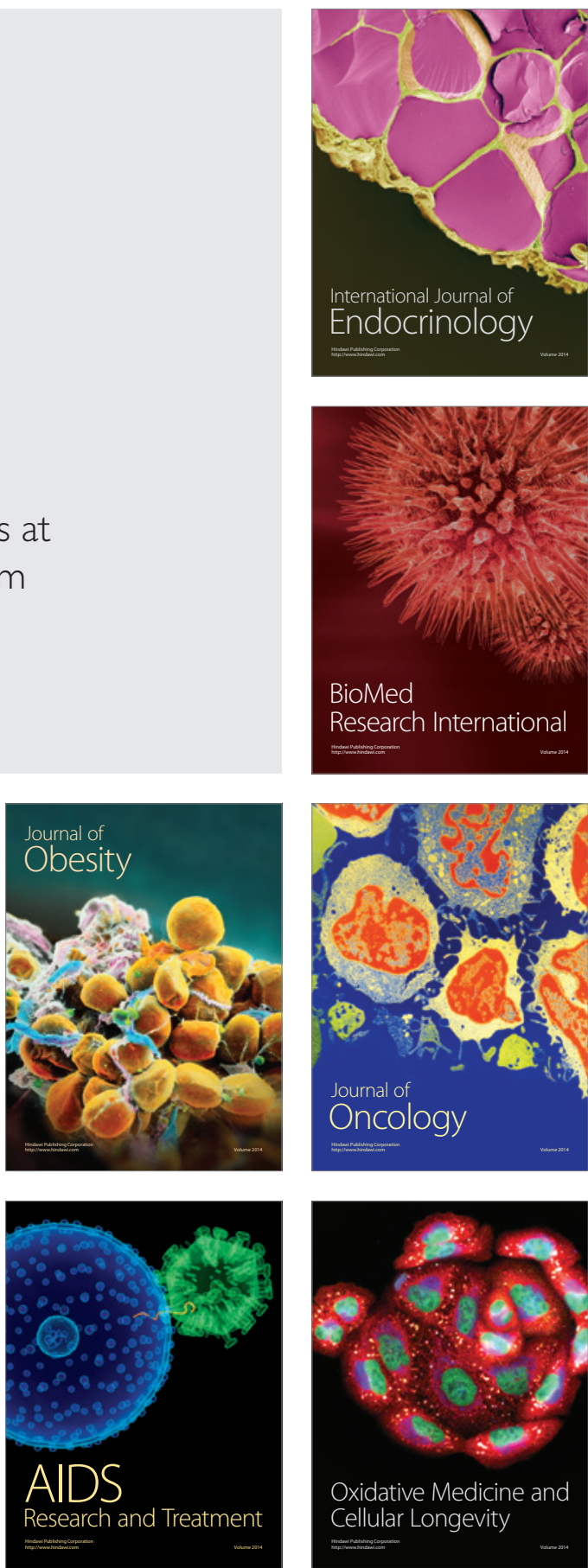